\title{
Agricultura tRAdicionAl Y RIQUEZA de MAÍCES (ZEA MAYS). Estudio de Caso en Caspalá, provincia de Jujuy, Argentina
}

\author{
RITA S. RAMOS ${ }^{1,2}$, NORMA I. HILGERT ${ }^{1,3}$ y DANIELA A. LAMBARÉ ${ }^{1,4}$
}

\begin{abstract}
Summary: Traditional agriculture and the richness of maize (Zea mays). A case study in Caspala, Jujuy province, Argentina. In Caspalá traditional corn landraces, and more recent ones, are cultivated. The current richness is the result of use, assessment, and management of this resource. This assessment is, in turn, associated with family decisions that reflect their culture, tradition and worldview. In this paper we analyze the richness of currently grown corn in Caspalá, the changes occurred in recent years, the use of the produce and management of agricultural land available. Semistructured interviews were conducted at $37(51 \%)$ families in the community. 23 ethnotaxa were reported (15 currently grown and 8 remembered), with an average of 6.61 maize ethnotaxa cultivated by family. The uses of each ethnotaxon, the growing in different microenvironments and the provenance of the "seeds", were identified as factors that positively influence the richness of corn crop. It was also found that the abandonment of transhumance, the decline of agriculture in different altitudes and the incorporation of foreign food products contributing to the decline of such richness. The results found warn about the importance of Caspalá, in the preserving of local maize, and the need to take steps for preserving existing richness.
\end{abstract}

Key words: Landraces, ethnobotany, transhumance.

Resumen: En Caspalá, se cultivan maíces criollos tradicionales y maíces de incorporación más reciente. La riqueza actual es producto del uso, valoración y manejo de este recurso. Dicha valoración está, a su vez, asociada a las decisiones familiares que reflejan su cultura, tradición y cosmovisión. En este trabajo se analiza la riqueza de maíces cultivados actualmente en Caspalá, los cambios ocurridos en los últimos años, el uso de lo producido y el manejo del espacio agrícola disponible. Se realizaron entrevistas semiestructuradas a $37(51 \%)$ familias de la comunidad. Se reportaron 23 etnotaxa (15 cultivados actualmente y 8 recordados), con un promedio de 6,61 etnotaxa sembrados por familia. Se identificaron como factores que influyen de modo positivo la riqueza de este cultivo los usos asignados a cada etnotaxón, el cultivo en diferentes microambientes y la procedencia de las "semillas". Asimismo se halló que el abandono de la actividad trashumante, la disminución de la actividad agrícola en distintos pisos altitudinales y la incorporación de productos alimenticios foráneos contribuyen a la disminución de tal riqueza. Los resultados hallados alertan sobre la importancia de Caspalá como conservador local de maíces y sobre la necesidad de desarrollar acciones que contribuyan a preservar las características actuales.

Palabras clave: Cultivos locales, etnobotánica, trashumancia.

\footnotetext{
${ }^{1}$ Consejo Nacional de Investigaciones Científicas y Técnicas.

${ }^{2}$ Centro de Investigaciones Científicas y Transferencia de Tecnología a la Producción (CICyTTP-CONICET). Diamante, Entre Ríos, Argentina.

${ }^{3}$ Instituto de Biología Subtropical. Facultad de Ciencias Forestales, Universidad Nacional de Misiones. Asoc. Civil Centro de Investigaciones del Bosque Atlántico. Bertoni 85, 3370, Puerto Iguazú, Misiones, Argentina. normahilgert@yahoo. com.ar

${ }^{4}$ Laboratorio de Botánica Sistemática y Etnobotánica. Facultad de Ciencias Agrarias. Universidad Nacional de Jujuy. San
} 


\section{INTRODUCCIÓN}

La productividad agrícola industrial, basada en el desarrollo y mantenimiento de cultivares mejorados, depende en parte de la disponibilidad de los recursos genéticos de poblaciones silvestres y/o de poblaciones donde los procesos de selección o domesticación siguen vigentes. En ese contexto la diversidad biológica y la diversidad sociocultural forman parte del desarrollo sostenible. Entendiendo como diversidad biológica a la variabilidad genética y fenotípica existente, para un organismo dado, a nivel molecular, individual, poblacional y ecosistémico (Gepts, 2004) y como diversidad sociocultural a aquella definida por los conocimientos y percepciones de los pueblos (Wood \& Lenné, 1999).

Para el caso del maíz, es posible diferenciar variedades modernas desarrolladas -por técnicas de fitomejoramiento o ingeniería genética- con intereses en los cultivos industriales y variedades y/o razas locales. Estas últimas, son aquellas generadas a partir de la selección natural y artificial y adaptadas al manejo y a las características locales en concordancia con la definición propuesta por Aguirre Gómez et al. (1998). Dichas variedades locales, en la literatura fueron denominadas landraces (Louette et al., 1997), etnovariedades o variedades criollas (Perales et al., 2005). En el presente trabajo emplearemos el apelativo etnotaxa (Hilgert et al., 2013), de modo de incluir todas las categorías taxonómicas (subespecies, variedades, razas e híbridos).

En el caso del maíz, la constante selección, producto de la sapiencia y las necesidades de los pueblos, ha generado la diversificación en razas locales (etnotaxa o landraces) en gran parte de las áreas donde se cultiva de modo familiar (Hernández, 1985; Pressoir \& Berthaud, 2004, Perales et al., 2003; Cámara Hernández et al., 2012). Hacia el fin del siglo XX y principios del actual, más de la mitad de la superficie de maíz cultivada en las regiones templadas de los países en vías de desarrollo, aún provenía de "semillas" producidas y seleccionadas por el propio agricultor (Morris, 2002), siendo México el principal referente (Bellon \& Berthaud, 2004). En el presente Argentina ocupa el segundo lugar como productora a nivel mundial de maíces transgénicos; cultivo que supera en superficie a las variedades locales (http://www.isaaa.org/
resources/publications/briefs/37/pptslides/GlobalStatus-Map-2007.pdf). No obstante, dentro del territorio argentino hay regiones en las que, desde tiempos remotos y hasta el presente, se siguen cultivando maíces nativos en sistemas agrícolas con técnicas tradicionales (Holmberg, 1904; Parodi, 1948; Cárdenas, 1949; Hilgert, 2007a; Cámara Hernández et al., 2012). Tal como se ha propuesto para otras regiones (Horovitz, 1935; Lia et al., 2007), la conservación de la diversidad de dichos maíces nativos depende de la acción que generan los manejos locales, así como de la conservación de los usos y valores específicos asociados a la cultura, tradición y cosmovisión de sus productores (Sturzenegger, 1982; Hilgert, 2004).

Enlaregión andina argentina, los maíces cultivados en el ámbito doméstico provienen de "semillas" (término empleado localmente para referirse a los frutos) de la cosecha anterior, combinadas con "semillas" obtenidas del intercambio con vecinos, entregadas por asesores de planes de desarrollo social, y/o adquiridas en mercados regionales (Hilgert, 1998; Hilgert \& Gil, 2005). Éste material genético es manejado y seleccionado según las técnicas tradicionales; con lo que se mantienen los etnotaxa locales, se adaptan o "criollizan" las foráneas y se generan nuevos etnotaxa, producto de combinaciones buscadas o accidentales. Estos procesos se asemejan a los descriptos para otras regiones de Latinoamérica (Louette et al., 1997; Louette \& Smale, 2000; Bellon \& Risopoulos, 2001; Perales et al., 2003). En ese contexto los estudios etnobotánicos cobran especial interés, dado que su abordaje ofrece información valiosa que puede contribuir al desarrollo de estrategias de conservación in situ, a partir del conocimiento y promoción del mantenimiento de agroecosistemas bajo manejo tradicional (Alcorn, 1984; Brush, 2000; Jarvis et al., 2000; Tuxill \& Nabhan, 2001).

En relación al uso y manejo del espacio, en regiones geográficas ambiental y culturalmente próximas al área de estudio, se ha observado que la trashumancia - la que implica el uso de diferentes pisos altitudinales y la permanencia en ellos - fomenta una mayor diversidad de recursos empleados, el cultivo de etnotaxa propios de cada ambiente y de otros más versátiles, así como la conservación de técnicas de manejo adecuadas a las características de cada sitio (Hilgert, 1998; Hilgert \& Gil, 2005; Hilgert, 2007b). 


\section{R. S. Ramos et al. - Agricultura tradicional y riqueza de maíces en Caspalá}

La comunidad de Caspalá representa un escenario tradicional, con pocas influencias urbanas modernas, diferente grado de conservación de la trashumancia y un relativo aislamiento geográfico (Ramos, 2009). Características similares han sido identificadas en regiones aledañas como contribuyentes al mantenimiento de la riqueza agrícola local (Hilgert \& Gil, 2006, 2008). Asimismo, en el presente la comunidad es identificada en la región como proveedora de "semillas" de maíces criollos de buena calidad, en particular algunos etnotaxa de tierras altas y actualmente poco cultivados. Estas características motivaron el estudio de los etnotaxa de maíces cultivados, en el pasado y el presente, los usos actuales de cada uno y el manejo del espacio agrícola disponible.

\section{Materiales y Métodos}

Se realizaron 3 campañas durante el año 2008: en abril, mayo (época de cosecha) y agosto (momento de seleccionar las "semillas" para cultivar en el ciclo agrícola siguiente), totalizando 30 días de trabajo. Durante las mismas se efectuaron entrevistas semiestructuradas y observación participante en la comunidad como parte de la convivencia durante las estadías. La elección de los entrevistados se realizó al azar, entre los que se trabajó con aquellos que consintieron colaborar con el estudio. Dado que en su seno se deciden y realizan las actividades relacionadas al cultivo del maíz, la unidad de muestreo fue la familia nuclear. Se trabajó con 37 familias $(51 \%)$ del total de familias que integran la comunidad). La edad de los jefes de cada unidad nuclear osciló entre 24 y 86 años; el grupo etario más representado en el muestreo fue el de 60 a 69 años (39\% de la muestra), seguido del grupo de los de 30 a 39 años (27\%), los de 40 a 49 y 50 a 59 años (ambos 13\% de la muestra) y finalmente el grupo de los de 70 a79 años con el $8 \%$ restante.

Durante las entrevistas se indagó acerca de: los etnotaxa cultivados, la totalidad de apelativos regionales para cada uno, sus respectivos tiempos de maduración y los sitios preferenciales de cultivo. Asimismo se registraron los usos asignados, la pérdida o cambio del elenco de etnotaxa cultivados por la familia a lo largo del tiempo, y el destino de la producción. Los datos fueron registrados en cuadernos de campo y grabaciones de voz en soporte de audio. Una vez completadas las entrevistas se visitaron las parcelas con maíces en condiciones de ser cosechados, los sitios de acondicionamiento ("pela de mazorcas") antes del almacenamiento. En la época de seleccionar las "semillas" para sembrar en el nuevo ciclo productivo, se analizó junto con los propietarios el contenido de las cestas de almacenamiento.

Se colectaron un total de 109 muestras de maíces (Tabla 1) de los diferentes etnotaxa cultivados. Cada muestra comprende entre 3 a 8 espigas, según disponibilidad de material del informante. En todos los casos, el material fue seleccionado por el productor, usando el mismo criterio que si estuviese eligiendo "semillas" para la próxima siembra. El acondicionamiento de las muestras estuvo a cargo de R. Soledad Ramos (SR), quien además las identificó con la orientación del Ing. Jorge Schimpf, tomando en cuenta el trabajo de Cámara Hernández et al. (2012). Posteriormente, parte de este material fue revisado por taxónomos del Banco de Germoplasma de Pergamino y por el Ing. Cámara Hernández, de la Facultad de Agronomía de la UBA. Dichas muestras fueron depositadas en el Banco de Germoplasma de INTA Pergamino, el resto del material está depositado en el herbario de referencia del Laboratorio de Botánica Sistemática y Etnobotánica de la Facultad de Ciencias Agraria de la UNJu.

Los registros de voz y los cuadernos de campo se conservan en el Laboratorio de Botánica Sistemática y Etnobotánica de la Facultad de Ciencias Agraria de la UNJu. El registro de las muestras se encuentra en el catálogo personal de la primera autora con la asignación RS seguido por el número de colección. En este aporte denominamos etnotaxa a las poblaciones de maíces distinguidas por los agricultores, en concordancia con el concepto de landraces propuesto por Perales et al. (2003). A dichos etnotaxa, además de nombre vernáculo, se les reconocen localmente caracteres particulares (como color, propiedades culinarias y/o medicinales, tamaño de la planta). Teniendo en cuenta el marco teórico de las etnociencias antes expuesto, los etnotaxa Overo, Colorado, Charrochillo y Bolita, son considerados aquí como una entidad taxonómica, dado que en las entrevistas hubo uniformidad de criterio a la hora de seleccionarlos, denominarlos y emplearlos. De los preparados culinarios citados en el presente trabajo 
se pueden hallar mayores detalles en Hilgert (1999) y Cámara Hernández \& Arancibia de Cabezas (2007).

En relación a la riqueza de etnotaxa cultivados, se compararon las identificaciones vernáculas y las taxonómicas, se estimó el promedio y el desvío estándar de etnotaxa cultivados por familia. Con respecto al patrón de siembra de los distintos etnotaxa según los microclimas o ambientes, se diagramaron los distintos sitios de cultivos dentro de los ambientes reconocidos (Fig. 1) y se comparó el uso de los mismos según los etnotaxa que cultiva cada familia. Para ello se tuvo en cuenta la totalidad de etnotaxa sembrados por microambiente, por un lado, y solo aquellas que llegan a madurar, por el otro. Para estas comparaciones se realizaron test de Chi cuadrado.

\section{Resultados}

\section{Características Socioculturales de Caspalá}

La población local está compuesta mayoritariamente por adultos y niños pequeños (en general los jóvenes se trasladan en busca de alternativas laborales). Son criollos nativos, conservadores de las costumbres, tradiciones e idiosincrasia local. Hablan castellano enriquecido con vocablos quechuas. El poblado cuenta con una escuela primaria de jornada simple. En el año 2008 se inauguró la extensión de la ruta provincial $\mathrm{N}^{\circ} 73$, que comunica Caspalá con los pueblos vecinos. Esta vía rápida facilita la comunicación $\mathrm{y}$, con ello, es probable que en los próximos años se registren cambios en la dinámica local e interregional.

Se observa una economía de subsistencia, donde las principales actividades son la agricultura y la ganadería para consumo local, complementadas ocasionalmente con trabajos asalariados y con trabajos transitorios en la zafra azucarera o en cosechas de frutales en el sur del país (manzanas, vides, entre otros). Según la precepción local, la producción ganadera ha disminuido en los últimos años debido al abandono paulatino de la trashumancia y la sedentarización de las familias. Aproximadamente el $25 \%$ de la población cuenta con un empleo en el municipio local y parte de los mayores percibe una jubilación, debido a su pasado trabajo en el Ingenio Azucarero Ledesma.
En el municipio se plantaron sauces (Salix humboldtiana Willd.) y álamos (Populus sp.) con el objeto de frenar los vientos, disminuir el efecto de las heladas y aportar madera (para la construcción de las casas y cercos y para leña). Según los pobladores, desde el establecimiento de estas cortinas de viento, el microclima se tornó paulatinamente más templado y permitió el cultivo de especies que prosperan en ambientes ubicados a menor altura respecto del nivel del mar. No obstante, dadas las diferentes exposiciones de los sitios de cultivos y las diferentes alturas en Caspalá se reconoce un microgradiente climático este-oeste que modela la riqueza de cultivos en cada sector. En zonas altas se cultivan mayoritariamente papas (Solanum tuberosum L.) y ocas (Oxalis tuberosa Mol.) y en las zonas bajas maíces y otros cultivos incorporados recientemente. Todas las familias de Caspalá practican la agricultura, en distintas proporciones y con diferente rol en la economía familiar.

Según los testimonios de los mayores, desde épocas remotas se cultiva maíz, papa, porotos (Phaseolus vulgaris L.), papa lisa (Ullucus tuberosus Loz.) y oca. Con las influencias post hispánicas se incorporaron nuevas especies como trigo (Triticum aestivum L.), haba (Vicia faba L.), arveja (Pisum sativum L.) y cebada (Hordeum vulgare L.). Más recientemente se enriquecieron las huertas con numerosas hortalizas, entre ellas acelga (Beta vulgaris L. var. cicla), repollo (Brassica oleracea L.) y lechuga (Cichorium endivia L., Lactuca sativa L.). No obstante, los cultivos más importantes en la comunidad son la papa y el maíz. Entonces, al momento de explicar las formas de cultivar, fue constante la comparación, por parte de los informantes, entre estos dos productos. Por ejemplo: “...la papa requiere de más cuidado que el maíz, necesita suficiente abono, es muy importante el riego antes de la siembra para evitar la entrada al tubérculo del llamado gusano lacato (larva de coleóptero). En cambio el maíz es más "obediente", es decir necesita menos cuidado, pero sí mucho riego para que apure a madurar y no ingrese a la mazorca el gusano del choclo.....".

\section{Riqueza de maices}

Se reportaron 23 etnotaxa, 15 cultivados actualmente y 8 recordados, comprendidos en 9 


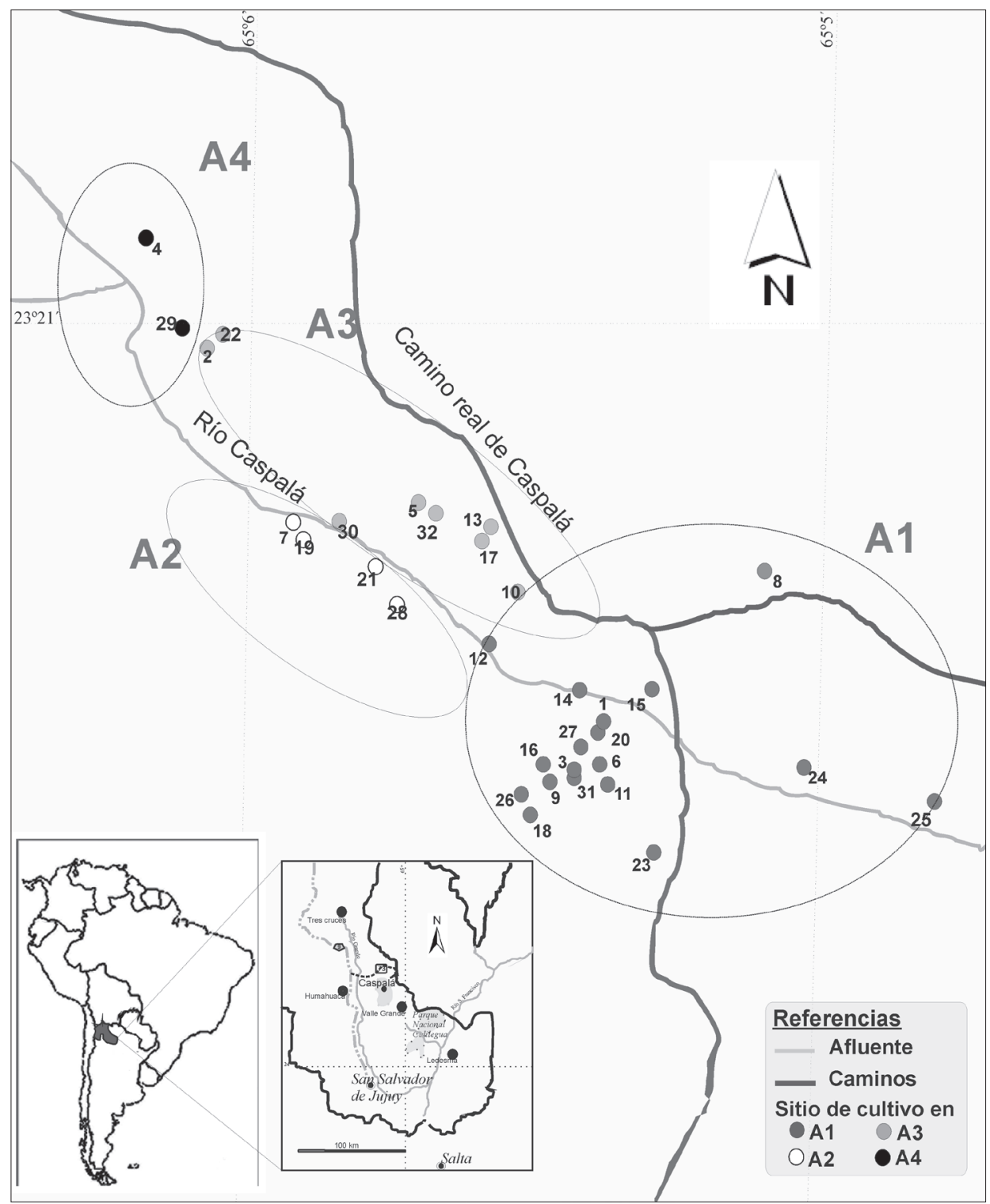

Fig. 1. Área de estudio y ubicación de los sitios de cultivo de las familias entrevistadas. $A 1=$ parte central del poblado (2900 y 3100 m.s.n.m.), la zona más apta para la actividad agrícola con riego, tanto al aire libre como en invernáculos. A2 = ladera con exposición este (3000 a los 3100 m.s.n.m.) con cortinas de viento y riego. $\mathrm{A} 3$ = ladera con exposición sudoeste (3060 a 3190 m.s.n.m), similar a la anterior pero con menor tiempo de luz solar y calor. A4 = sitio ubicado a unos 4 km del poblado (3180 a los 3220 m.s.n.m), con pendientes de 30 a $45 \%$ y suelos arenosos.

razas puras y 6 mezcladas o hibridadas (según Cámara Hernández in litt. y Cámara Hernández et al., 2012) y en tres variedades de la especie, Zea mays var. indurata (con 2 razas y 3 mezclas), $Z$. mays var. amylacea (con 6 razas y 3 mezclas) y $Z$. mays var. oryzaea (con 1 raza) (según el equipo de especialistas del Banco de Germoplasma de Pergamino -Schlatter in litt.-). Los 8 etnotaxa recordados incluyen maíces que han dejado de cultivarse en el transcurso de los últimos 20 años 
aproximadamente. Para incluirlos en la región se consideraron sólo aquellos etnotaxa conocidos por el productor que los citó y cultivados, en un pasado reciente, por él mismo o por sus padres. Teniendo en cuenta la complejidad de la taxonomía de esta especie, tanto en el sistema folclórico como en el formal, en adelante nos referiremos a 15 etnotaxa, considerando a las mezclas como entidades taxonómicas. El promedio de etnotaxa cultivados por familias es de 6,61 con un desvío estándar de 2,56 , siendo 1 la menor cantidad hallada en una familia y 13 la mayor. En la Tabla 1 se incluye la totalidad de nombres vernáculos registrados para cada etnotaxón, se observa que algunos recibieron hasta doce apelativos distintos. En algunos casos el nombre empleado es aquel que porta tradicionalmente ese etnotaxón de maíz; en otros más que un denominativo es un apelativo descriptivo (en particular cuando se trata de etnotaxa que se mezclaron) o el nombre que usaba quien le proveyó la "semilla" al actual usuario.

En relación al empleo de lo producido, la mayor parte es para consumo hogareño, aunque también se intercambia con vecinos, con productores de otras comunidades, o se vende cuando es necesario contar con dinero. Dentro del consumo hogareño, se emplea como forraje, alimento y, en menor medida, con fines medicinales (Tabla 1).

El maíz, en el área de estudio, va perdiendo importancia como alimento básico debido al reemplazo parcial por productos de mercado, como arroz y fideo. No obstante, los precios de estos productos foráneos siguen siendo elevados para la economía local, por lo que aún se consume diariamente "mote" (grano de maíz hervido entero, con o sin el pericarpo) y frecuentemente

Tabla 1. Diversidad de Maíces de Caspalá, ciclo de vida y usos asignados.

\begin{tabular}{|c|c|c|c|c|c|c|}
\hline $\begin{array}{l}\text { Nombre } \\
\text { Vernáculo } \\
\text { Unificado ( }{ }^{\circ} \\
\text { colección) }\end{array}$ & Nombre Vernáculo & Variedad $^{1}$ & $\begin{array}{c}\text { Raza Pura } \\
\text { (mezcla o } \\
\text { hibridación) }^{2}\end{array}$ & $\begin{array}{c}\mathrm{N}^{\circ} \text { de } \\
\text { Reportes } \\
\text { (cultivado/no } \\
\text { cultivado en } \\
\text { el presente) }\end{array}$ & $\begin{array}{l}\text { Usos } \\
\text { reportados }\end{array}$ & $\begin{array}{l}\text { Ciclo de } \\
\text { vida }^{3}\end{array}$ \\
\hline $\begin{array}{l}\text { Amarillo Chico } \\
\text { (RS 1; 10; 14; } \\
\text { 17; 35; 49; 66; } \\
\text { 74\#; 78; 81; } \\
86 ; 88 ; 109^{\#)}\end{array}$ & $\begin{array}{l}\text { Amarillo Chico, } \\
\text { Amarillo, A. } 8 \text { Rayas }\end{array}$ & indurata & Amarillo Chico & $19 \backslash 1$ & $\begin{array}{l}\text { Chicha, } \\
\text { anchi, mote } \\
\text { pela, choclo } \\
\text { y humitas }\end{array}$ & Largo $^{4}$ \\
\hline $\begin{array}{l}\text { Amarillo } \\
\text { Grande (RS } \\
11 ; 18 ; 42 ; 54 ; \\
56 ; 73 ; 84^{\# ;} \\
\left.85^{\#} ; 90 ; 99\right)\end{array}$ & $\begin{array}{l}\text { Amarillo Ancho, } \\
\text { Anaranjado, Amarillo } \\
\text { Jujeño, Amarillo Claro, } \\
\text { Amarillo Grande, } \\
\text { Capia Marlo Negro }\end{array}$ & indurata & $\begin{array}{c}\text { (Amarillo } \\
\text { Chico x } \\
\text { Harinoso) }\end{array}$ & $21 \backslash 1$ & $\begin{array}{l}\text { Chicha, } \\
\text { anchi, mote } \\
\text { pela, choclo, } \\
\text { humitas; para } \\
\text { los animales }\end{array}$ & Largo \\
\hline $\begin{array}{l}\text { Blanco (RS 5; } \\
7 ; 27 ; 28 ; 41 ; \\
53 ; 61 ; 65 ; 79 ; \\
92 ; 97 ; 101)\end{array}$ & $\begin{array}{l}\text { Blanco Capia, Capia, } \\
\text { Diente de Caballo, } \\
\text { Blanco Grande, Blanco } \\
\text { Tarijeño, Cambial }\end{array}$ & amylacea & Capia & 3813 & $\begin{array}{l}\text { Harina para } \\
\text { tortillas } \\
\text { y bollos, } \\
\text { humitas, } \\
\text { choclo }\end{array}$ & Largo \\
\hline $\begin{array}{l}\text { Bolita (RS 15; } \\
22 ; 52 ; 55 ; 57 \\
63 ; 77 ; 83 ; 91 ; \\
95 \# ; 97 ; 100)\end{array}$ & $\begin{array}{l}\text { Bolita, Morochito, Bola } \\
\text { Chiquito, Bolita Amarillo, } \\
\text { Bola Blanquito, Bola } \\
\text { Blanco, Bola Amarillo, } \\
\text { Bolita Morocho, Blanco } \\
\text { Bolita, Bolita Chiquito, } \\
\text { Amarillo Bola Chiquito, } \\
\text { Amarillo Chico }\end{array}$ & indurata & $\begin{array}{l}\text { (Altiplano } \\
\text { mezcla) }\end{array}$ & 3212 & $\begin{array}{l}\text { Harina } \\
\text { cocida para } \\
\text { ulpada, sanco } \\
\text { y chilcán; } \\
\text { tijtincha, } \\
\text { choclo y } \\
\text { humitas, para } \\
\text { los animales }\end{array}$ & Corto $^{4}$ \\
\hline
\end{tabular}




\section{R. S. Ramos et al. - Agricultura tradicional y riqueza de maíces en Caspalá}

\begin{tabular}{|c|c|c|c|c|c|c|}
\hline $\begin{array}{l}\text { Nombre } \\
\text { Vernáculo } \\
\text { Unificado (N} \\
\text { colección) }\end{array}$ & Nombre Vernáculo & Variedad $^{1}$ & $\begin{array}{c}\text { Raza Pura } \\
\text { (mezcla o } \\
\text { hibridación) }{ }^{2}\end{array}$ & $\begin{array}{l}\text { No de } \\
\text { Reportes } \\
\text { (cultivado/no } \\
\text { cultivado en } \\
\text { el presente) }\end{array}$ & $\begin{array}{l}\text { Usos } \\
\text { reportados }\end{array}$ & $\begin{array}{l}\text { Ciclo de } \\
\text { vida }^{3}\end{array}$ \\
\hline $\begin{array}{l}\text { Canario (RS } \\
29 ; 75 ; 82^{\# ;} \\
94^{\# ;} 96^{\#} ; \\
103^{\# ;} \text { 104) }\end{array}$ & $\begin{array}{l}\text { Marlo Chico, Amarillo } \\
\text { Delgadito, Amarillo } \\
\text { Finito, Chiquito, } \\
\text { Marlo Finito }\end{array}$ & amylacea & Chaucha & $10 \backslash 1$ & $\begin{array}{l}\text { Harina tostada } \\
\text { dulce para } \\
\text { ulpada y } \\
\text { chilcán }\end{array}$ & Corto \\
\hline Capia (RS 76) & Capia Marlo Negro & amylacea & Harinoso & 110 & $\begin{array}{l}\text { Harina } \\
\text { para bollos } \\
\text { y tortillas; } \\
\text { choclo, } \\
\text { humitas, mote, } \\
\text { tijtincha }\end{array}$ & $\begin{array}{l}\text { Intermedio } \\
\text { a largo }\end{array}$ \\
\hline $\begin{array}{l}\text { Capia Rosado } \\
\text { (RS 6; 8; } \\
\left.34 ; 106^{\#}\right)\end{array}$ & Capia Rosado, Rosadito & amylacea & Capia Rosado & $7 \backslash 4$ & $\begin{array}{l}\text { Harina } \\
\text { para bollos } \\
\text { y tortillas, } \\
\text { choclo, humita, } \\
\text { mote, tijtincha }\end{array}$ & Largo \\
\hline $\begin{array}{l}\text { Charrochillo } \\
\text { (RS 26; } \\
30 ; 44 * 50 ; \\
59 ; 64)\end{array}$ & $\begin{array}{l}\text { Charrochillo, Capia } \\
\text { Charrochillo, Rayadito, } \\
\text { Chorreado }\end{array}$ & indurata & $\begin{array}{c}\text { (Morocho } \\
\text { Variegado) }\end{array}$ & 813 & $\begin{array}{l}\text { Frangollada, } \\
\text { mote, tijtincha, } \\
\text { para los } \\
\text { animales }\end{array}$ & Largo \\
\hline $\begin{array}{l}\text { Colorado (RS } \\
\text { 12; 20; 33\#; } \\
\text { 43; 46"; 89; } \\
93 ; 107)\end{array}$ & $\begin{array}{l}\text { Colorado, Rojo, } \\
\text { Coloradito }\end{array}$ & amylacea & $\begin{array}{l}\text { (Colorado con } \\
\text { pericarpio } \\
\text { púrpura) }\end{array}$ & 510 & $\begin{array}{l}\text { Chicha, anchi, } \\
\text { choclo }\end{array}$ & Largo \\
\hline $\begin{array}{l}\text { Culli }(4 ; 16 ; \\
31 ; 58 ; 87)\end{array}$ & $\begin{array}{l}\text { Culli, Culli chico, } \\
\text { Culli grande }\end{array}$ & amylacea & Culli & $17 \backslash 2$ & $\begin{array}{l}\text { Chicha, anchi, } \\
\text { api5, medicinal } \\
\text { (para limpias); } \\
\text { mágico } \\
\text { (protege las } \\
\text { chacras) }\end{array}$ & $\operatorname{Largo}^{4}$ \\
\hline $\begin{array}{l}\text { Garrapata } \\
\text { (RS 21; 23; } \\
\text { 25\#; 37; 45"; } \\
\left.62 ; 98 ; 102^{\#}\right)\end{array}$ & $\begin{array}{l}\text { Garrapatillo, Garrapata, } \\
\text { Okesito, Capia } \\
\text { Garrapata, Oke, } \\
\text { Garrapata Overito, } \\
\text { Garrapatillo Blanco, } \\
\text { Garrapatillo Rosado }\end{array}$ & amylacea & Garrapata & $24 \backslash 1$ & $\begin{array}{l}\text { Harina tostada } \\
\text { para chilcán y, } \\
\text { ulpada; choclo } \\
\text { y humita }\end{array}$ & $\begin{array}{l}\text { Intermedio/ } \\
\text { largo } 4\end{array}$ \\
\hline $\begin{array}{l}\text { Macanacho } \\
\text { (RS 24; 32\#; } \\
36 ; 48 ; 70)\end{array}$ & Macanacho, Camacho & indurata & $\begin{array}{l}\text { (Morocho } \\
\text { cruza con } \\
\text { Marrón y } \\
\text { Garrapata) }\end{array}$ & $1 \backslash 1$ & $\begin{array}{l}\text { Chicha, anchi, } \\
\text { frangollada; } \\
\text { harina para } \\
\text { bollos y } \\
\text { tortillas; para } \\
\text { los animales }\end{array}$ & $\begin{array}{l}\text { Largo a } \\
\text { intermedio }\end{array}$ \\
\hline $\begin{array}{l}\text { Morocho (RS } \\
2 ; 9 ; 13 ; 38 ; \\
39 ; 47^{\# ;} 51 ; 67 ; \\
\left.68 ; 69^{\# ;} ; 80\right)\end{array}$ & $\begin{array}{l}\text { Morocho, Morochito } \\
\text { Amarillo, Morocho } \\
\text { Blanco, Bola Morocho } \\
\text { Blanco, Perla }\end{array}$ & indurata & Morocho & 2516 & $\begin{array}{l}\text { Frangollada, } \\
\text { sopa majau, } \\
\text { chicha, anchi; } \\
\text { para los } \\
\text { animales }\end{array}$ & Corto \\
\hline
\end{tabular}


Bol. Soc. Argent. Bot. 48 (3-4) 2013

\begin{tabular}{|c|c|c|c|c|c|c|}
\hline $\begin{array}{c}\text { Nombre } \\
\text { Vernáculo } \\
\text { Unificado ( }{ }^{\circ} \\
\text { colección) }\end{array}$ & Nombre Vernáculo & Variedad $^{1}$ & $\begin{array}{c}\text { Raza Pura } \\
\text { (mezcla o } \\
\text { hibridación) }{ }^{2}\end{array}$ & $\begin{array}{c}\mathrm{N}^{\circ} \text { de } \\
\text { Reportes } \\
\text { (cultivado/no } \\
\text { cultivado en } \\
\text { el presente) }\end{array}$ & $\begin{array}{c}\text { Usos } \\
\text { reportados }\end{array}$ & $\begin{array}{c}\text { Ciclo de } \\
\text { vida }^{3}\end{array}$ \\
\hline $\begin{array}{l}\text { Overo (RS 71; } \\
\left.72 ; 105 ; 108^{\#}\right)\end{array}$ & Overo, Overo Capia & amylacea & $\begin{array}{c}\text { (Harinoso, } \\
\text { mezcla) }\end{array}$ & 810 & $\begin{array}{l}\text { Harina } \\
\text { para bollos } \\
\text { y tortillas; } \\
\text { choclo, } \\
\text { humitas }\end{array}$ & $\begin{array}{l}\text { Intermedio } \\
\text { a largo }{ }^{4}\end{array}$ \\
\hline $\begin{array}{l}\text { Pisincho (RS } \\
3 ; 19 ; 40 ; 60)\end{array}$ & $\begin{array}{l}\text { Pisincho Espinudito, } \\
\text { Pisincho Overo, } \\
\text { Pisingallo, Pisincho } \\
\text { Amarillo, Pisincho } \\
\text { Blanco }\end{array}$ & oryzaea & Pisingallo & 2316 & $\begin{array}{l}\text { Harina tostada } \\
\text { para chilcán } \\
\text { y ulpada; } \\
\text { pochochos }\end{array}$ & largo \\
\hline Bolita Chiquito & & & no colectado & 015 & $\begin{array}{l}\text { Harina para } \\
\text { ulpada y } \\
\text { chilcán }\end{array}$ & \\
\hline $\begin{array}{l}\text { Chiquito } \\
\text { para Cavar }\end{array}$ & Para Cavar & & no colectado & 013 & $\begin{array}{l}\text { Harina para } \\
\text { ulpada y } \\
\text { chilcán }\end{array}$ & \\
\hline Chuipe $^{6}$ & Chulpe & & no colectado & $0 \backslash 1$ & $\begin{array}{l}\text { Choclo, } \\
\text { humitas; } \\
\text { harina tostada } \\
\text { para ulpada } \\
\text { y chilcán }\end{array}$ & \\
\hline Naranjita & & & no colectado & $0 \backslash 1$ & $\begin{array}{l}\text { Harina tostada } \\
\text { para chilcán } \\
\text { y ulpada }\end{array}$ & \\
\hline $\begin{array}{l}\text { Bola Grande } \\
\text { Blanco }\end{array}$ & & & no colectado & $0 / 1$ & $\begin{array}{l}\text { Mote, harina } \\
\text { para bollos } \\
\text { y tortillas; } \\
\text { tijtincha }\end{array}$ & \\
\hline Colorado ${ }^{7}$ & & & no colectado & $0 \backslash 1$ & Chicha, anchi & \\
\hline $\begin{array}{l}\text { Capia } \\
\text { Medianito }\end{array}$ & & & no colectado & $0 \backslash 1$ & $\begin{array}{l}\text { Harina tostada } \\
\text { para chilcán } \\
\text { y ulpada }\end{array}$ & \\
\hline Rojito Chico ${ }^{8}$ & & & no colectado & $0 \backslash 1$ & $\begin{array}{l}\text { Harina tostada } \\
\text { para chilcán } \\
\text { y ulpada }\end{array}$ & \\
\hline
\end{tabular}

\footnotetext{
1 según especialistas del Banco de Germoplasma Activo de Maíz, Estación Experimental Agropecuaria Pergamino; ${ }^{2}$ según Cámara Hernández et al 2012. Se señala con \# el material revisado por el especialista; ${ }^{3}$ ciclo de vida largo: octubre-mayo; Corto: octubre- febrero/noviembre-marzo; intermedio: octubre-abril; ${ }^{4}$ previador, es decir que produce choclos antes que el resto de los entotaxa cultivados en simultáneo; ${ }^{5}$ se hierve la harina obtenida con clavo de olor y jugo de limón [Citrus $\times$ limon (L.) Osbeck.] o de naranja agria (Citrus $\times$ aurantium L.) , se endulza y se consume generalmente caliente; ${ }^{6}$ según las descripciones y los usos citados se trata de la raza Chullpi; ${ }^{7}$ probablemente se trate de la raza Colorado; ${ }^{8}$ probablemente se trate de la raza Capia Púrpura.
} 


\section{R. S. Ramos et al. - Agricultura tradicional y riqueza de maíces en Caspalá}

"tijtincha" (mazorca de maíz hervida luego de quitar algunas hileras de granos). Al usarlo como forraje se emplean las mazorcas de algunos etnotaxa en especial y las plantas secas de todo lo cosechado. Estas últimas se guardan, como reserva amontonadas en los predios de cultivos para las épocas en que los pastos escasean. Por otra parte, numerosas familias señalan que uno de los principales objetivos a la hora de la siembra del maíz es reproducir y mantener algunos etnotaxa locales poco cultivados $\mathrm{y}$, por ende, difíciles de hallar. Esto último coincide con lo expresado por los agricultores de la región al citar frecuentemente a Caspalá como una localidad "proveedora" de "semillas" de buena calidad y de etnotaxa tradicionales.

En la Tabla 1 se señala el ciclo de vida de cada etnotaxón. Según la clasificación realizada por los productores, en líneas generales, los maíces grandes (con espigas grandes) tienen un ciclo de vida largo -es decir tardan en madurar alrededor de 7 meses de ciclo vegetativo- y los maíces chicos (con espigas chicas) presentan un ciclo de vida corto- -es decir alcanzan la madurez entre los 4 y 5 meses de ciclo vegetativo-. Entre ambos extremos se conciben etnotaxa con ciclos de vida intermedios capaces de madurar unos 5 o 6 meses de ciclo vegetativo.

El 48,35\% de los entrevistados nombró etnotaxa de maíces que alguna vez sembraron y que actualmente, por distintas razones (como pérdida de la "semilla", cambios en las preferencias culinarias, etc.), ya no lo hacen. En este sentido, la escasez - o la ausencia- de un mercado que demande los etnotaxa tradicionales empleados para la culinaria típica son unas de las principales limitaciones citadas a la hora de justificar el abandono del cultivo. Cada familia, en promedio, citó entre 2 y 3 etnotaxa que no cultivan en el presente. En relación al tiempo que hace que cada etnotaxón se cultiva en la comunidad, se han señalado como bien adaptadas a la altura a todas los etnotaxa recordados (Chiquito para Cavar, Chuipe, Naranjita, Bola Grande Blanco, Colorado, Capia medianito y Rojito), y a otros actuales como Amarillo Chico, Bolita, Canario, Culli, Garrapata, Macanacho, Morocho, Overo y Pisincho.

Respecto a los etnotaxa recordados, el Maíz para Cavar y el Chuipe probablemente han desaparecido de la comunidad, puesto que no se han hallado reportes de su cultivo actual en ninguna familia.
Los mismos, según el discurso local, poseen características muy peculiares: desarrollan plantas pequeñas y con mazorcas también pequeñas, dispuestas al ras del suelo y granos muy dulces. Estos etnotaxa en la actualidad prácticamente se convirtieron en un mito de los agricultores. Se los consideran difíciles de cultivar, dado que presentan un bajo rendimiento en relación a la energía que se invierte en su producción.

El conjunto de lo expresado oficia de justificativo, o explicación, de su reemplazo por nuevos etnotaxa. Estos nuevos etnotaxa, a su vez, se pudieron incluir en la producción local gracias al paulatino incremento de la temperatura de la zona en las últimas décadas (debido principalmente a las modificaciones antes descriptas realizadas para ello).

En cuanto al estado actual de cultivo de cada etnotaxón, teniendo en cuenta la cantidad de familias que los siguen cultivando y la superficie destinada a cada uno a lo largo del tiempo, se consideró en una situación intermedia (entre el abandono y la conservación) a los maíces Bolita, Canario, Macanacho, Capia, Overo y Culli. De éstos, el Bolita está conceptuado como uno de los mejores para preparar comida pero, dado su bajo rendimiento y su abandono en la culinaria local, su producción decae años tras año. Del mismo modo el maíz Pisingallo se cultiva cada vez menos, lo que se fundamenta en dos aspectos: 1) es un maíz "rogado", es decir que necesita mucha atención hasta su madurez, y 2) los granos tienen terminaciones punzantes (son puntiagudos) que lastiman las manos al desgranar la mazorca. Finalmente, los maíces Canario y Macanacho son desplazados en el presente por maíces grandes (de ciclo de vida largo) incorporados recientemente por su mayor rendimiento.

Dentro de los maíces "foráneos" los etnotaxa Colorado, Blanco y Amarillo Grande son los más recientes en la zona. Los tres son muy apreciados por el tamaño de sus mazorcas y por su productividad. Se incorporaron al elenco local después de las modificaciones ambientales antes descritas, dado que son susceptibles al frío y la sequía. Dentro del manejo reportado, se mencionó que las "semillas" de estos etnotaxones recientemente incorporados, deben ser renovadas por materiales provenientes de otros sitos cada dos o tres años, de lo contrario decaen la calidad y la productividad. Las espigas de los maíces Blanco y Colorado se consumen 
como choclo y para elaborar harinas para panes, en tanto que el Amarillo Grande se incorporó por sus bondades como forrajera.

\section{Los maíces en Caspalá y los sitios de cultivo}

De acuerdo con las características de relieve, pendiente, exposición al sol y al viento, disponibilidad de agua y amplitud térmica diaria, los productores de la región identifican 4 zonas distintas -denominadas ambientes en este trabajo-. La Fig. 1 señala la ubicación y amplitud de cada uno de esos ambientes donde las familias informantes tienen sus sitios de cultivo. En la Tabla 2 se señala el número de citas de los etnotaxa sembrados en cada ambiente y en la Fig. 2 se representa el porcentaje relativo de sitios utilizados para cultivar cada etnotaxón.

El ambiente que abarca la parte central del poblado (A1) se ubica entre los 2900 y 3100 m.s.n.m., es un valle alto protegido ("encajonado") por altos cerros con exposición hacia el noreste, lo que garantiza una prolongada exposición solar y vientos suaves y templados. El mismo es reconocido como la zona más apta para la actividad agrícola, tanto al aire libre como en invernáculos. En concordancia con esto, allí cultiva el mayor número de familias (23 de las 37 entrevistadas). Esta zona, presenta canales de riego funcionales, con una distribución equitativa del agua. En relación con la actividad agrícola, el único aspecto desfavorable mencionado para esta zona es que el crecimiento del pueblo compite con el espacio apto para cultivar.

Otro de los espacios agrícolas (A2), se ubica desde los 3000 a los 3100 m.s.n.m., y está formado por terrenos con clima templado durante las mañanas y, por lo general, con vientos vespertinos fríos y secos, lo que define una vegetación típica de ambientes puneños. La superficie de este sector y el número de familias que lo cultiva son menores

Tabla 2. Número de citas de los maíces cultivados en cada ambiente. A1 = parte central del poblado (2900 y 3100 m.s.n.m.), la zona más apta para la actividad agrícola con riego, tanto al aire libre como en invernáculos. A2 = ladera con exposición este (3000 a los 3100 m.s.n.m.) con cortinas de viento y riego. A3 = ladera con exposición sudoeste (3060 a 3190 m.s.n.m), similar a la anterior pero con menor tiempo de luz solar y calor. A4 = sitio ubicado a unos 4 km del poblado (3180 a los 3220 m.s.n.m), con pendientes de 30 a $45 \%$ y suelos arenosos.

\begin{tabular}{|lccccc|}
\hline & Ambiente 1 & Ambiente 2 & Ambiente 3 & Ambiente 4 & TOTAL \\
Amarillo Chico & 9 & 5 & 5 & 0 & 19 \\
Amarillo Grande & 14 & 4 & 3 & 0 & 21 \\
Blanco & 23 & 10 & 5 & 0 & 38 \\
Bolita & 18 & 4 & 8 & 2 & 32 \\
Canario & 4 & 2 & 3 & 1 & 10 \\
Capia & 0 & 0 & 1 & 0 & 1 \\
Capia Rosado & 4 & 3 & 0 & 0 & 7 \\
Charrochillo & 5 & 2 & 1 & 0 & 8 \\
Colorado & 3 & 2 & 0 & 0 & 5 \\
Culli & 8 & 4 & 5 & 0 & 17 \\
Garrapata & 17 & 2 & 4 & 1 & 24 \\
Macanacho & 1 & 0 & 0 & 0 & 1 \\
Morocho & 10 & 7 & 6 & 2 & 25 \\
Overo & 2 & 2 & 4 & 0 & 8 \\
Pisincho & 12 & 7 & 3 & 1 & 23 \\
Total & 130 & 54 & 47 & 7 & 239 \\
\hline
\end{tabular}




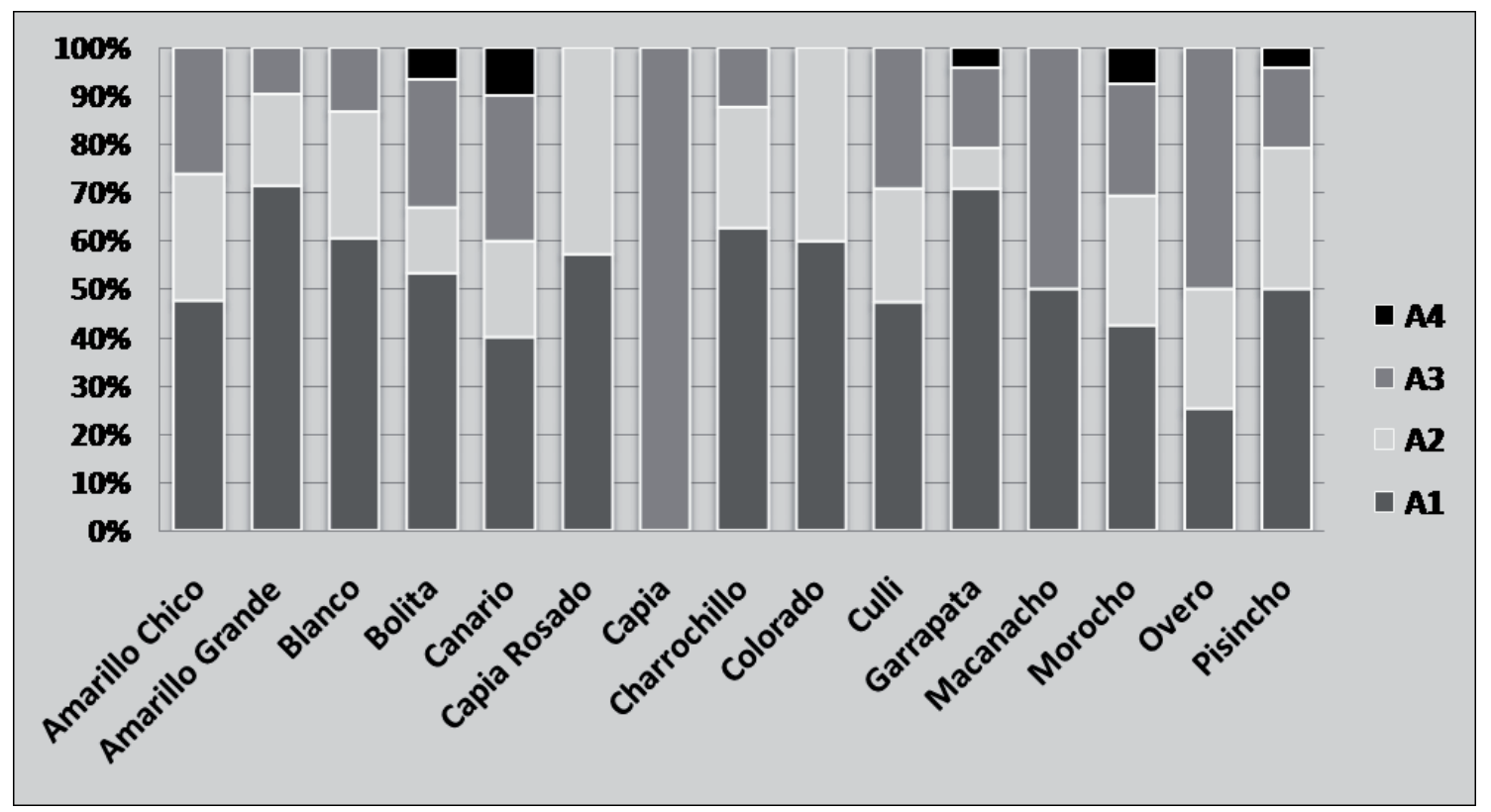

Fig. 2. Porcentaje relativo de los ambientes donde se cultiva cada etnotaxa. Ver referencias en Fig. 1.

que en el anterior ( 6 familias de las 37 analizadas tienen una parcela aquí). El maíz prospera aquí gracias a las cortinas de viento plantadas hace unos 50 años (antes descriptas) y al riego. Este último se practica mediante canales que distribuyen agua de vertientes locales. Dichas vertientes se ubican en quebradas de la región; en su mayor parte es agua de los deshielos diarios. Dado que esta zona es muy fría para algunos maíces, los etnotaxa que más se adaptan son los maíces chicos. No obstante, también se suelen cultivar los maíces grandes, pero, generalmente, en cantidades menores y se destinan al consumo de las espigas inmaduras (como choclo), ya que las probabilidades de que maduren antes de las heladas otoñales son bajas y, si lo logran, la calidad del producto no es buena.

Un tercer sector dedicado a la agricultura (A3) corresponde a la ladera con exposición sudoeste, por lo que cuenta con poca irradiación solar diaria. Se emplaza a una altura que va desde los 3060 a 3190 m.s.n.m. Nueve de las familias entrevistadas cultivan aquí. En este ambiente también hay cortinas de vientos y el criterio de uso es similar al del ambiente anterior. El agua para riego también proviene de las quebradas cercanas, pero, dado el menor aporte de calor diario en otoño e invierno las vertientes se mantienen congeladas disminuyendo así su disponibilidad. Para contrarrestar este efecto construyeron un sistema de canales más extenso, con el que se trae, además, agua del río principal. No obstante, no siempre se logra una distribución adecuada y, por lo tanto, se cultiva menos en estas laderas. Otro problema, identificado en esta área es que las crecidas recurrentes y extraordinarias del río Caspalá, van erosionando parte de la superficie destinada a la producción y periódicamente interrumpen los canales de riego construidos.

Finalmente, un espacio ubicado a una altura que va desde los 3180 a los 3220 m.s.n.m y que presenta pendientes muy pronunciadas (de 30 a 45\%), constituye el cuarto ambiente reconocido (A4). En este sector los suelos son poco fértiles y arenosos, lo que exige un adecuado manejo de abonos y de rotación de cultivos. También aquí hay disponibles canales de riego con agua proveniente de las quebradas subyacentes y del río principal. Es el sitio más alejado del pueblo central (a unos $4 \mathrm{~km}$ ). Se entrevistaron a 2 familias que cultivan en este ambiente y es donde se observó el menor número de etnotaxa cultivados, dado que sólo prosperan 
aquellos que se adaptan a la altura y al frío (en particular los maíces Bolita y Canario). Como caso excepcional, una de las familias visitadas siembra en este ambiente maíz Garrapata, el que se consume como choclo.

Las familias que viven en el ambiente 4 (A4) cultivan allí solo maíces chicos, al resto lo cultivan en el ambiente A1, o lo obtienen por medio de trueques o compra con familias que producen en los otros ambientes (Tabla 2).

Teniendo en cuenta la totalidad de los etnotaxa sembrados en los distintos ambientes, no se observa un uso diferencial de los mismos $\left(\chi^{2}{ }_{36 \text { g.l. }}\right.$, $\mathrm{p}=0.745=30,09)$. Sin embargo, cuando se consideran sólo los etnotaxa que llegan a madurar en cada ambiente, sí hay una diferencia significativa $\left(\chi_{36}^{2}\right.$ g.l.' $\left.{ }_{p=0,002}=65,18\right)$, resultando el Área 1 el diferencial, pues cuando éste es excluido del análisis desaparece dicha divergencia $\left(\chi_{24 \text { g.l. }{ }^{2}=0,59}=21,81\right)$.

\section{Discusión y Conclusiones}

Dentro de los maíces actuales, el discurso de los pobladores respecto a la presencia antigua del etnotaxón Capia concuerda con lo mencionado por Cámara Hernández et al. (2012), quienes proponen que esta raza se habría originado en el noroeste argentino. En la misma obra se sostiene que el Amarillo Chico y el Harinoso habrían originado al Amarillo Grande. Este último es de incorporación reciente al cultivo local y muy apreciado por sus características; sus "semillas" provienen de mercados de las urbes vecinas.

De acuerdo al testimonio de los productores locales, los maíces Bolita, Garrapata, Macanacho, Culli, Charrochillo, Canario son etnotaxa típicos en la agricultura de las familias en Caspalá, dado que los mismos son parte del patrimonio conservado a través de las generaciones. A la vez, a los etnotaxa Bolita, Garrapata y Culli se los considera localmente como originarios de Bolivia. Esto podría deberse a antiguos intercambios entre habitantes de ambos países, situaciones comunes en el pasado de acuerdo a diversos autores (Ventura, 1995; Nuñez \& Dillehay, 1995; Albeck, 2001; Yacobaccio et al., 1999). El maíz Culli es considerado un etnotaxón que comparte presencia en el territorio boliviano y en las provincias argentinas de Jujuy y Salta (Cámara Hernández et al., 2012). Su distribución, probablemente está asociada a su importancia en la cosmovisión y la medicina local (Hilgert, 1998, 2001; Ramos, 2009 Hilgert et al., 2013).

En el presente aporte, se registra la presencia de razas no citadas antes para la provincia de Jujuy. El etnotaxón Canario (raza Chaucha según Cámara Hernández et al., 2012), extiende el área de distribución de este cultivar, el que había sido antes citado exclusivamente para Salta. Del mismo modo, el maíz Colorado (de RS33 y RS46), identificado por Cámara Hernández como "Colorado con pericarpio púrpura", posiblemente se trate de la raza Capia púrpura, cultivada actualmente solo en algunas áreas de Jujuy y Salta (Cámara Hernández in litt.).

Los etnotaxa Overo, Macanacho y Charrochillo fueron reconocidos por los taxónomos como mezclas. En concordancia con esto, el maíz Overo es considerado localmente como una mezcla originada a partir de la falta de un intercambio de "semillas" entre vecinos, por un manejo inadecuado de las parcelas o porque no se separan las "semillas" durante el almacenamiento.

Dentro de los maíces recordados el maíz Chuipe por las descripciones pareciera hacer referencia al maíz Chullpi. Según los taxónomos, podría ser producto de la cruza entre Capia y el Chullpi típico, dado que ambas razas presentan una floración tardía sincrónica (Cámara Hernández et al., 2012). El maíz Chullpi típico fue descrito por dos autores para la misma región, Grobman et al. (1961) sostiene que es pariente de una raza de origen peruano cultivada en sitios cercanos en el pasado, y que su distribución alcanzó territorio argentino en épocas del Imperio Incaico, la otra se describe para la raza cultivada en Bolivia y la Quebrada de Humahuaca, en Argentina (Brieger et al., 1958).

En este trabajo se pone en evidencia la plasticidad genotípica de la especie, a través de la diversidad fenotípica de los etnotaxa presentes en la región, de la mano de estrategias de manejo y decisiones de conservación. En la bibliografía es común hallar citados factores que atentan contra la conservación de la diversidad del maíz; entre ellos se mencionan el desuso en la culinaria (Hilgert, 1998; Cámara Hernández \& Arancibia de Cabezas, 2007; Cámara Hernández et al., 2012), el abandono de las curas rituales (Forgione, 1982; Pérez de Nucci, 1988; Ibarra Grasso, 1999; Hilgert, 2004; Pelegrin, 2005) y la preferencia de los agricultores por etnotaxa 


\section{R. S. Ramos et al. - Agricultura tradicional y riqueza de maíces en Caspalá}

más grandes y con mayor productividad que casi siempre corresponde a maíces introducidos o foráneos. Lo observado en Caspalá podría considerarse el comienzo de este proceso, donde la gente mayor recuerda, y aún prepara, las recetas culinarias de sus antecesores, pero en lo cotidiano el maíz va siendo reemplazado por el arroz y los fideos. Si se mantiene esta tendencia en el marco de modificaciones culturales, es esperable que las generaciones jóvenes abandonen el uso y el cultivo de algunos etnotaxa locales.

Según lo observado, en la actualidad no necesariamente se registra una disminución de la riqueza, sino una modificación del elenco de etnotaxa que la componen. Dichos cambios, se sustentan en un profundo conocimiento del ecosistema local y de cómo manipularlo, de modo de adaptarlo a los requerimientos de los cultivos recientemente incorporados. Estas modificaciones se generan principalmente por la actual necesidad de las familias de transformar la economía de autoconsumo por una que le provea divisas. Esto que probablemente conducirá, en un futuro próximo, a una reducción de la riqueza de maíces a nivel macro regional por la desaparición de los etnotaxa raros o exclusivos de estos ambientes.

En concordancia con lo que se propone en la Convención sobre Conservación de la Biodiversidad (Glowka et al., 1994), es importante que se generen acciones de un modo ágil y consensuado con los propios agricultores, a fin de desarrollar estrategias de conservación in situ que garanticen la pervivencia de los conocimientos y del material genético local. En otras regiones hay numerosos ejemplos de este tipo de acciones (Milpa Project, 1999; Bellon et al., 2003). Fundamentalmente, es importante que dichas respuestas contribuyan a revertir el abandono de la vida rural y de la agricultura como sistema productivo. Este fenómeno ya fue descripto en otras regiones, en particular cuando los que deciden son jóvenes con formación académica y contacto con ciudades (Bellon \& Berthaud, 2004).

Por otra parte, respecto a la nomenclatura local, la diversidad de apelativos hallados probablemente se explique al analizar en profundidad los factores asociados a la adquisición y transmisión del conocimiento y de las "semillas". Es probable que en ocasiones el productor desconozca el nombre del etnotaxón que está cultivando y frente a la pregunta haya improvisado un apelativo descriptivo. De todos modos, este tema es complejo y sería necesario explorarlo con mayor profundidad para comprenderlo.

El hecho que el ambiente "menos natural", o más manipulado, producto de un largo proceso de acondicionamiento (con cortinas de viento y riego), sea el único que presentó diferencias significativas en cuanto a lo que allí se cultiva, puede interpretarse como evidencia del conocimiento local de esta especie. Este conocimiento está fundamentado en la plasticidad de la misma y en la acción seleccionadora del hombre modelada con el aprovechamiento altitudinal de la región, hecho este último documentado en numerosas ocasiones (Camino, 1982; Ventura, 1995; Reboratti, 1996, 1997; Casas et al., 1997; Ibarra Grasso, 1999).

En síntesis, la riqueza de maíces presente en la comunidad de Caspalá, el estado de conservación de las características culturales propias de este pueblo y el uso vigente y cotidiano del maíz en la alimentación familiar, muestran un panorama aún alentador para promocionar y resguardar la riqueza del germoplasma del lugar. Algunos cambios recientes (como la apertura del camino carretero y la introducción de maíces híbridos) podrían influenciar negativamente en la conservación de algunos etnotaxa en particular. Consideramos que para resguardar esta comunidad es fundamental respetar y valorar sus particularidades y estimular la economía local generando estrategias de mercado para sus productos tradicionales. Posiblemente éste sea un método efectivo para revigorizar el interés por producir sus propios maíces, sin la necesidad de incorporar nuevos.

\section{Agradecimientos}

A la comunidad de Caspalá, por la valiosa cooperación y participación brindada durante el desarrollo del trabajo. En particular a la Señora Lucía y al Hospital de Humahuaca y a la Comisión Municipal por facilitar la logística en las campañas. A los niños y maestros de Caspalá por la compañía. A Dora Vignale por contribuir a nuestro encuentro y por su cariñoso apoyo profesional y humano. A Fernanda Fabbio, Fernando Zamudio, Guillermo Gil, Jorge Schimpf, Lauro Cazón, Dante Hormigo, Julián Cámara Hernández, Silvia Abarza y Alejandro Casas por su ayuda desinteresada en diferentes 
etapas de esta tarea. Este trabajo se realizó con apoyo económico de la Red RISAPRET/ Programa Iberoamericano CYTED y el PIP 191 (CONICET).

\section{Bibliografía}

AGUIRRE GÓMEZ, J. A., M. BELLON \& M. SMALE. 1998. A regional analysis of maize biological diversity in Southeastern Guanajuato, Mexico. Econ. Work. Paper 98 (06): 1-16.

ALBECK, M. 2001. La Puna Argentina en los períodos medios y tardíos. En: Berberián \& Nielsen (Eds.), Historia Argentina Prehispánica, pp. 347-388. Ed. Brujas, Córdoba.

ALCORN, J. B. 1984. Huastec mayan ethnobotany. University of Texas Press, Austin.

BELLON, M. R. \& J. RISOPOULOS. 2001. Small-scale farmers expand the benefits of improved maize germplasm: a case study from Chiapas, Mexico. World Dev. 29: 799-811.

BELLON, M. R., J. BERTHAUD, M. SMALE, J. A. AGUIRRE, S. TABA, F. ARAGÓN, J. DÍAZ \& H. CASTRO. 2003. Participatory landrace selection for on farm conservation: an example from the Central Valleys of Oaxaca, Mexico. Genet. Resour. Crop Evol. 50: 401-416.

BELLON, M. R. \& J. BERTHAUD. 2004. Transgenic maize and the evolution of landrace diversity in Mexico. The importance of farmers' behavior. Plant Physiol. 134: 883-888.

BRIEGER, F. G., J. T. GURGEL, E. PATERNIANI \& M. R. ALLEONI. 1958. Races of Maize in Brazil and other Eastern South American countries. NAS-NRC Publ. N 593.

BRUSH, S. B. 2000. The issues of in situ conservation of crop genetic resources. In: S. B. Brush (ed.), Genes in the Field: On-farm conservation of crop diversity. pp. 3-26. IPGRI, IDRC, Lewis Publishers, Rome, Ottawa, Boca Raton.

CÁMARA HERNÁNDEZ, J. \& D. ARANCIBIA DE CABEZAS. 2007. Maíces andinos y sus usos en la Quebrada de Humahuaca y regiones vecinas. Editorial Facultad de Agronomía, UBA, Buenos Aires.

CÁMARA HERNÁNDEZ, J., A. M. MIANTE ALZOGARAY, R. BELLÓN \& J. A. GALMARINI. 2012. Razas de maíz nativas de la Argentina. Editorial Facultad de Agronomía, UBA, Buenos Aires.

CAMINO, A. 1982. Tiempo y espacio en la estrategia de subsistencia andina: un caso en las vertientes orientales Sud-Peruanas. Senri Ethnolog. Studies 10: 11-37.
CÁRDENAS, M. 1949. Plantas alimenticias nativas de los Andes. Cereales y otras semillas. Folia Universitaria (Cochabamba) 3: 102-119.

CASAS A., J. CABALLERO, C. MAPES \& S. ZÁRATE. 1997. Manejo de la vegetación, domesticación de plantas y origen de la Agricultura en Mesoamérica. Bol. Soc. Bot. México 61: 31-47.

FORGIONE, C. A. 1982. Estudio antropológico cultural de la sociedad rural de la Quebrada de Humahuaca. Tesis doctoral, Facultad de Filosofía y Letras, UBA.

GEPTS, P. 2004. ¿Who owns biodiversity and how should the owners be compensated?. Plant Physiol. 134: 1295-1307.

GLOWKA, L., F. BURHENNE-GUILMIN \& H. SYNGE. 1994. A guide to the convention on biological diversity. IUCN, Gland and Cambridge.

GROBMAN A., W. SALHUANA \& R. SEVILLA. 1961. Races of maize in Peru. NAS-NRC Publ. No 915.

HERNÁNDEZ, E. 1985. Maize and the greater southwest. Econ. Bot. 39: 416-430.

HILGERT, N. I. 1998. Las plantas vinculadas con el ámbito doméstico y la subsistencia de los campesinos de la cuenca del río Zenta, Dpto. Orán, Prov. Salta. Tesis Doctoral, Facultad de Ciencias Exactas, Físicas y Naturales, UNC.

HILGERT, N. I. 1999. Las plantas comestibles en una zona de las Yungas meridionales (Argentina). Anal. Jardin Bot. Madrid, 57: 117-138.

HILGERT, N. I. 2001. Plants used in home medicine in the Blanco River basin, in northwestern Argentina subtropical montane forests. J. Ethnopharm. 76: 11-34.

HILGERT, N. I. 2004. Las plantas en las festividades religiosas de la selva andina argentina. Bull. Soc. suisse Américan. 68: 37-49.

HILGERT, N. I. 2007a. La vinculación del hombre actual con los recursos naturales y el uso de la tierra. En: A.D. Brown, M. García Moritán, B.N. Ventura, N.I. Hilgert \& L.R. Malizia (eds.), Finca San Andrés. Un Espacio de Cambios Ambientales y Sociales en el Alto Bermejo. pp. 159-186. Ed. del Subtrópico, Tucumán.

HILGERT, N. I. 2007b. Plantas silvestres, ámbito doméstico y subsistencia. En: A.D. Brown, M. García Moritán, B.N. Ventura, N.I. Hilgert \& L.R. Malizia (eds.), Finca San Andrés. Un Espacio de Cambios Ambientales y Sociales en el Alto Bermejo, pp. 187-228. Ed. del Subtrópico, Tucumán.

HILGERT, N. I. \& G. E. GIL. 2005. Traditional Andean agriculture and changing processes in the Zenta river basin, Salta, Northwestern Argentine. Darwiniana 43: 30-43.

HILGERT, N. I. \& G. E. GIL. 2006. Medicinal plants of the Argentine Yungas plants of the Las Yungas biosphere reserve, Northwest of Argentina, used in 


\section{R. S. Ramos et al. - Agricultura tradicional y riqueza de maíces en Caspalá}

health care. Biod. Conserv. 15: 2565-2594.

HILGERT, N. I. \& G. E. GIL. 2008. Los cambios de uso del ambiente y la medicina herbolaria. Estudio de Caso en Yungas Argentinas. Blacpma 7: 130-140.

HILGERT, N. I., F. ZAMUDIO, V. FURLAN \& L. CARIOLA. 2013. The key role of cultural preservation in maize diversity conservation in the Argentine Yungas. Evidence-Based Complementary and Alternative Medicine. Article ID 732760, 10 pages. Doi: 10.1155/2013/732760.

HOLMBERG, E. A. 1904. Investigación agrícola de la Provincia de Jujuy. Anales del Ministerio de Agricultura. Sec. Agr., Bot. Agr. 2: 138-150.

HOROVITZ, S. 1935. Distribución geográfica de los factores genéticos en los maíces autóctonos del norte argentino. Revista Argent. Agron. 2: 133-135.

IBARRA GRASSO, D. 1999. Tras las huellas del origen del maíz. Servicio Informativo Ibero Americano. OEI, La Paz.

JARVIS, D., B. STHAPIT \& L. SEARS. 2000. Conserving agricultural biodiversity in situ: a scientific basis for sustainable agriculture. International Plant Genetic Resources Institute, Rome.

LIA, V. V., V. A. CONFALONIERI, N. RATTO, J. A. CÁMARA HERNÁNDEZ, A. M. MIANTE ALZOGARAY, L. POGGIO \& T. A. BROWN. 2007. Microsatellite typing of ancient maize: insights into the history of agriculture in southern South America. Proc. Biol. Sci. 274: 545-554.

LOUETTE, D., A. CHARRIER \& J. BERTHAUD. 1997. In situ conservation of maize in Mexico: genetic diversity and maize seed management in a traditional community. Econ. Bot. 51: 20-38.

LOUETTE, D. \& M. SMALE. 2000. Farmers' seed selection practices and traditional maize varieties in Cuzalapa, Mexico. Euphytica 113: 25-41.

MILPA PROJECT. 1999. Conservation of genetic diversity and improvement of crop production in Mexico: a farmer-based approach: 1999 Annual Report. http://www.grcp.ucdavis.edu/milpa.

MORRIS, M. L. 2002. Impacts of international maize breeding research in developing countries 1966-98. CIMMYT, Mexico. Disponible en: http://books. google.com.ar/books [Acceso: 20 Julio 2009].

NUÑEZ L. \& T. DILLEHAY. 1995. Movilidad giratoria, armonía social y desarrollo en los Andes Meridionales: Patrones de tráfico e interacción económica. Universidad Católica del Norte, Antofagasta.

PARODI, L. R. 1948. Los maíces indígenas de la República Argentina. Anal. Acad. Nac. Ci. Exactas 11: 9-14.

PERALES, H., S. B. BRUSH \& C. O. QUALSET. 2003. Dynamic management of maize landraces in central Mexico. Econ. Bot. 57: 21-34.
PRESSOIR, G. \& J. BERTHAUD. 2004. Population structure and strong divergent selection shape phenotypic diversification in maize landraces. Heredity 92: 95-101.

PELEGRIN, M. 2005. Cuando la salud viene de la tierra. Una visión antropológica de la medicina Popular en Jujuy. Buenos Aires, Del Umbral.

PÉREZ DE NUCCI, A. 1988. La Medicina Tradicional del Noroeste Argentino. Historia y Presente. Ed. del Sol, Buenos Aires.

RAMOS, R. S. 2009. Factores culturales, ambientales $y$ de manejo involucrados en la pérdida $y / o$ conservación de variedades criollas de maíces en la comunidad rural de Caspalá, Provincia de Jujuy. Tesina, Facultad de Ciencias Agrarias, Universidad Nacional de Jujuy.

REBORATTI, C. 1996. Sociedad, ambiente y desarrollo Regional en la Alta Cuenca del Río Bermejo. Instituto de Geografía, Facultad de Filosofía y Letras, UBA.

REBORATTI, C. (Comp.). 1997. De hombres y tierras. Una historia ambiental del Noroeste Argentino. Proyecto Desarrollo Agroforestal en Comunidades Rurales del Noroeste Argentino. Salta.

STURZENEGGER, O. 1982. Área de la selva TucumanoOranense, San Andrés: Actividades de subsistencia tradicionales y ritual propiciatorio. Documenta Laboris 27. Programa de Investigaciones sobre Epidemiología Psiquiátrica, Consejo Nacional de Investigaciones Científicas y Técnicas. Buenos Aires. Argentina.

TUXILL J. \& G. P. NABHAN. 2001. People, plants and protected areas. A guide to in situ management. Earthscan Publications Ltd., London.

VENTURA, B. N. 1995. Modelo preliminar de uso del espacio en los valles Orientales a las serranías de Zenta (Salta). En: A. D. Brown \& R. Grau (eds.), Investigación, Conservación y Desarrollo en Selvas Subtropicales de Montañas. pp. 191-198. Universidad Nacional de Tucumán, Tucumán.

WOOD, D. \& J. M. LENNÉ(eds.). 1999. Agrobiodiversity: characterization, utilization, and management. CABI, Wallingford.

YACOBACCIO H., P, ESCOLA, F. PEREYRA, M. GLASCOCK \& M. LAZZARI. 1999. Desde dónde y hacia dónde: Localización de fuentes y distribución de Obsidianas en el NOA. Resúmenes XIII Congreso Nacional de Arqueología Argentina, Córdoba.

Recibido el 16 de octubre de 2012, aceptado el 01 de agosto de 2013 . 
